\title{
Evaluation of novel urinary tract infection biomarkers in children
}

\author{
Joshua R. Watson', David S. Hains'², Daniel M. Cohen', John David Spencer', Jennifer M. Kline ${ }^{3}$, Han Yin ${ }^{4}$ and
} Andrew L. Schwaderer'

BACKGROUND: Pediatricians frequently use urinalysis to diagnose urinary tract infection (UTI) while awaiting urine culture results, but sensitivity and specificity of urinalysis are limited. This study evaluated the diagnostic accuracy of the antimicrobial peptides (AMPs) human $\alpha$-defensin 5 (HD5) and human neutrophil peptides (HNP) 1-3 as novel UTI biomarkers in children.

METHODS: We prospectively enrolled 199 pediatric Emergency Department or Urgent Care patients evaluated for a UTI. Urine concentrations of HD5 and HNP1-3 were measured by enzyme-linked immunosorbent assay. Urine culture was the reference standard. Sensitivities and specificities of leukocyte esterase (LE), HD5, HNP1-3, and test combinations were compared.

RESULTS: For predicting positive urine culture, the areas under the receiver-operating characteristic curves for $\mathrm{HD} 5$ and HNP1-3 were 0.86 (95\% confidence interval (Cl): 0.81-0.92) and 0.88 (95\% Cl: 0.82-0.93), respectively. Compared to LE $\geq$ trace, the combination test "LE and HD5" increased specificity by $6 \%$ (95\% Cl: 3-10\%) without decreasing sensitivity. In the subgroup whose urine was collected by a clean-catch method, combination tests "LE and HD5" and "HD5 and HNP13 " increased specificity by > 10\% compared to LE alone.

CONCLUSION: Urine AMP profiles are a promising novel strategy as an adjunct to urinalysis to aid UTI diagnosis in children.

$\mathbf{U}$ rinary tract infection (UTI) is a common and potentially severe infection that represents a major burden of healthcare utilization/expenditure and antibiotic exposure in children (1-3). The diagnosis of UTI relies on suggestive symptoms, pyuria, and isolation of a uropathogen in culture. However, UTI symptoms in children often are nonspecific (4), and culture results are not available at the time of initial evaluation. Consequently, providers frequently rely on rapid results of the urine dipstick to decide whether to initiate empiric antibiotic therapy for a presumed UTI while awaiting culture results. This approach may lead to either delayed diagnosis or unnecessary antibiotic therapy because of the limitations of leukocyte esterase (LE) and urinary nitrite on dipstick urinalysis. Specifically, large pediatric studies and meta-analyses of the utility of the urine dipstick for predicting positive urine culture have consistently shown suboptimal sensitivity and/or specificity of individual and combination tests, even though results of individual studies vary widely (5-8).

In this study, we evaluated two peptides in the $\alpha$-defensin family of antimicrobial peptides (AMPs) as novel biomarkers for UTI in children. AMPs are small, cationic peptides that participate in the innate immune defense of the kidneys, urinary tract, and other organ systems (9). AMPs are produced by white blood cells and/or epithelial cells and may be constitutively expressed or induced when pathogens enter the urinary tract (9). Human $\alpha$-defensin 5 (HD5) is an epithelial-derived AMP produced by intestinal Paneth cells, the female genital tract, and the uroepithelium (10-12). In a prior study of a small number of children, our research group demonstrated via western immunoblot and enzyme-linked immunosorbent assay that HD5 was detected in culture-positive but not in culture-negative urine samples (12). Human neutrophil peptides (HNP) $1-3$ are three closely related $\alpha$-defensins (collectively known as HNP1-3 and typically measured in aggregate) produced in promyelocytes and stored in the primary granules of neutrophils (9). Two prior studies of adults demonstrated increased urine concentrations of HNP1-3 during acute UTI (13) and HNP1 during chronic pyelonephritis (14). Given this prior data, we hypothesized that HD5 and HNP1-3, alone or as adjuncts to dipstick urinalysis, would improve diagnostic accuracy for UTI. Therefore, the objective of this study was to evaluate the performance of urine HD5 and HNP1-3 concentrations as biomarkers for positive urine culture in children.

\section{RESULTS \\ Study Population}

During the study period, urine samples were collected from 268 Emergency Department (ED) or Urgent Care (UC) patients who met inclusion criteria. Of those, 34 had received

\footnotetext{
The first two authors and the last author contributed equally to this work.

'Department of Pediatrics, Nationwide Children's Hospital, The Ohio State University, Columbus, Ohio, USA; ${ }^{2}$ Innate Immunity Translational Research Center, Children's Foundation Research Institute at Le Bonheur Children's Hospital, Memphis, Tennessee, USA; ${ }^{3}$ Division of Emergency Medicine, Nationwide Children's Hospital, Columbus, Ohio, USA; “Biostatistics Core, Nationwide Children's Hospital, Columbus, Ohio, USA. Correspondence: Andrew L. Schwaderer (schwaderer.5@osu.edu)

Received 21 October 2015; accepted 13 December 2015; advance online publication 16 March 2016. doi:10.1038/pr.2016.33
} 
Table 1. Demographic characteristics and documented clinical signs and symptoms of study population ${ }^{\mathrm{a}}$

\begin{tabular}{|c|c|c|c|c|}
\hline & $\begin{array}{l}\text { All patients } \\
(n=199)\end{array}$ & $\begin{array}{l}\text { Catheterization }{ }^{b} \\
\qquad(n=99)\end{array}$ & $\begin{array}{l}\text { Clean-catch } \\
(n=100)\end{array}$ & $\begin{array}{l}\text { Catheterization vs. } \\
\text { clean-catch, } P \text { value }^{d}\end{array}$ \\
\hline Age in years, median (IQR) & $5.0(1.2-12.8)$ & $1.2(0.4-3.9)$ & $10.0(5.4-14.6)$ & $<0.001$ \\
\hline Fever & $95(48)$ & $67(68)$ & $28(28)$ & $<0.001$ \\
\hline Abdominal pain & $71(36)$ & $18(18)$ & $53(53)$ & $<0.001$ \\
\hline Urinary urgency or incontinence & $13(7)$ & 0 & $13(13)$ & $<0.001$ \\
\hline Back/flank pain or CVA tenderness & $24(12)$ & $4(4)$ & $20(20)$ & $<0.001$ \\
\hline Vomiting & $59(30)$ & $34(34)$ & $25(25)$ & 0.16 \\
\hline$\geq 1$ of above signs/symptoms & $177(89)$ & $87(88)$ & $90(90)$ & 0.66 \\
\hline
\end{tabular}

CVA, costovertebral angle; IQR, interquartile range.

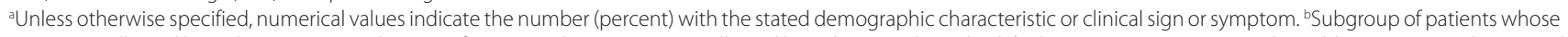
urine was collected by catheterization. `Subgroup of patients whose urine was collected by a clean-catch method. 'Fisher's exact test (categorical variables) or Mann-Whitney ranksum test (age).

Table 2. Sensitivities and specificities of LE and nitrite, alone and in combination

\begin{tabular}{|c|c|c|c|c|c|c|}
\hline \multirow[b]{2}{*}{ Test threshold } & \multicolumn{2}{|c|}{$\begin{array}{l}\text { All patients } \\
(n=199)\end{array}$} & \multicolumn{2}{|c|}{$\begin{array}{l}\text { Catheterization }{ }^{\mathrm{a}} \\
\quad(n=99)\end{array}$} & \multicolumn{2}{|c|}{$\begin{array}{c}\text { Clean-catch }^{b} \\
(n=100)\end{array}$} \\
\hline & $\mathrm{Se}, \%$ & $\mathrm{Sp}, \%$ & $\mathrm{Se}, \%$ & $\mathrm{Sp}, \%$ & $\mathrm{Se}, \%$ & $\mathrm{Sp}, \%$ \\
\hline $\mathrm{LE} \geq$ trace & 97 & 75 & 90 & 96 & 100 & 52 \\
\hline $\mathrm{LE} \geq$ small & 83 & 85 & 90 & 97 & 79 & 72 \\
\hline $\mathrm{LE} \geq$ moderate & 41 & 94 & 30 & 100 & 47 & 86 \\
\hline $\mathrm{LE}=$ large & 28 & 98 & 20 & 100 & 32 & 96 \\
\hline Nitrite & 45 & 99 & 40 & 99 & 47 & 99 \\
\hline $\mathrm{LE} \geq$ trace and nitrite & 45 & 99 & 40 & 99 & 47 & 99 \\
\hline $\mathrm{LE} \geq$ trace or nitrite & 97 & 75 & 90 & 96 & 100 & 52 \\
\hline
\end{tabular}

LE, leukocyte esterase; Se, sensitivity; Sp, specificity.

a Subgroup of patients whose urine was collected by catheterization. 's Subgroup of patients whose urine was collected by a clean-catch method.

antibiotics within $7 \mathrm{~d}$ before presentation and were excluded. Of the remaining 234 patients, 199 provided adequate excess urine sample volume for analysis of both HD5 and HNP1-3 concentrations. Table 1 describes the demographic characteristics and documented clinical signs and symptoms of the study population.

\section{Clinical Laboratory Results}

Of the 199 patients, 29 (15\%) had urine cultures that yielded $\geq 50,000$ colony-forming units/ $\mathrm{ml}$ of a single uropathogen. The positive urine cultures included $10(10 \%)$ of 99 in the subgroup of patients whose urine was collected by catheterization and $19(19 \%)$ of 100 in the subgroup whose urine was collected by a clean-catch method. Escherichia coli was the most common bacterial isolate, accounting for $25(86 \%)$ positive urine cultures, followed by Staphylococcus saprophyticus $(n=2)$, Klebsiella pneumoniae $(n=1)$, and Serratia marcescens $(n=1)$. Of the 29 patients with a positive urine culture, 23 (79\%) had signs or symptoms of upper UTI, including fever, back/flank pain, costovertebral angle tenderness, or vomiting. Table 2 lists the sensitivities and specificities of LE and nitrite, alone and in combination, for all patients and for subgroups by urine collection method.

\section{Antimicrobial Peptide Analyses}

Urine HD5 and HNP1-3 concentrations were significantly higher in culture-positive urine samples compared to culture-negative urine samples (Figure 1a,b). The median HD5 concentration was $590.6 \mathrm{pg}$ per $\mathrm{mg}$ creatinine $(\mathrm{pg} / \mathrm{mgCr}$ ) (interquartile range (IQR): 373.1-908.5) in culture-positive urine samples vs. $89.6 \mathrm{pg} / \mathrm{mgCr}$ (IQR: $12.8-253.5$ ) in culturenegative urine samples $(P<0.001$, Mann-Whitney). The median HNP1-3 concentration was $3,801.4 \mathrm{pg} / \mathrm{mgCr}$ (IQR: 1,776.6-11,861.3) in culture-positive urine samples vs. 148.7 $\mathrm{pg} / \mathrm{mgCr}$ (IQR: 25.7-934.2) in culture-negative urine samples $(P<0.001$, Mann-Whitney). The areas under the receiveroperating characteristic (ROC) curves for HD5 and HNP1-3 were 0.86 (95\% confidence interval (CI): 0.81-0.92) and 0.88 (95\% CI: 0.82-0.93), respectively (Figure 1c,d). Sensitivities and specificities of multiple test thresholds across the range of HD5 and HNP1-3 values are displayed in Supplementary Tables S1 and $\mathbf{S 2}$ online.

\section{Test Comparisons}

The sensitivity of the test "LE $\geq$ trace" was $97 \%$. HD5 and HNP1-3 thresholds of 174 and $384 \mathrm{pg} / \mathrm{mgCr}$, respectively, provided maximal specificity of each individual test with sensitivity at least equal to that of "LE $\geq$ trace." Comparisons of the sensitivities and specificities of LE, HD5, and HNP1-3, alone and in combination, for all patients are shown in Figure 2a. Nitrite was not included in these test comparisons due to its low sensitivity. The specificities of both HD5 and HNP1-3 were lower than the specificity of LE. However, the combination tests "LE and HD5" and "LE and HD5 and HNP1-3" increased specificity by $6 \%$ ( $95 \%$ CI: $3-10 \%)$ and $7 \%$ (95\% CI: $3-11 \%)$, respectively, compared to $\mathrm{LE}$ alone without decreasing sensitivity. 


\section{Articles | Watson et al.}
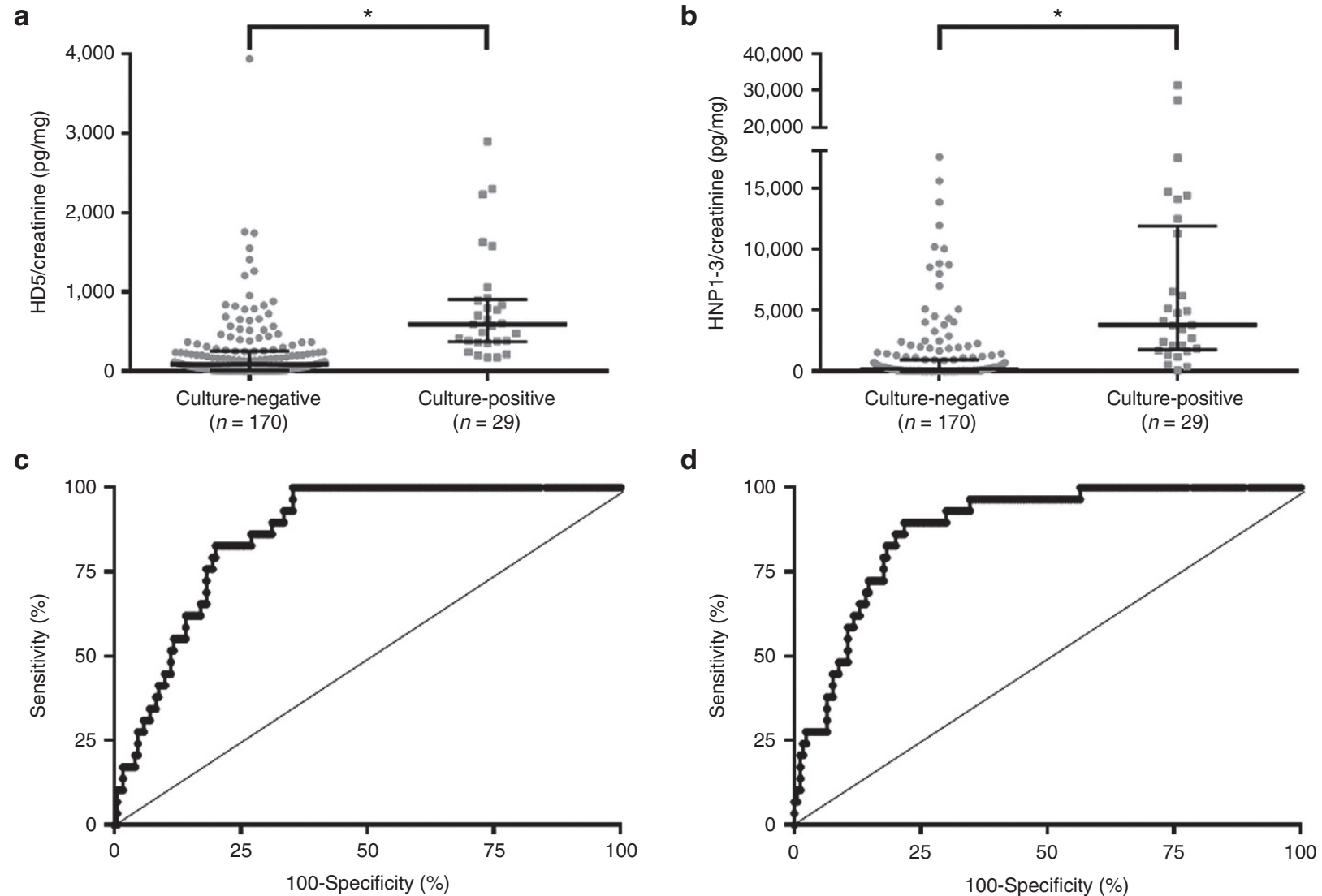

Figure 1. HD5 and HNP1-3 concentrations in culture-negative and culture-positive urine samples (a/b), and ROC curves (c/d). Horizontal bars represent median values and interquartile ranges for (a) HD5 and (b) HNP1-3. Gray circles and squares indicate individual data points. ROC curves for (c) HD5 and (d) HNP1-3 are presented. The diagonal line represents a test with no diagnostic value. ${ }^{*} P<0.001$. HD5, $\alpha$-defensin 5 ; HNP1-3, human neutrophil peptides $1-3$; ROC, receiver-operating characteristic.

\section{Analyses by Urine Collection Method}

Comparisons of LE, HD5, and HNP1-3 in the catheterization subgroup showed no increased specificity of combination tests vs. LE alone (Figure 2b). However, in the clean-catch subgroup, specificity increased 13\% (95\% CI: 6-21\%) when "LE and HD5" was compared to LE alone (Figure 2c). The combination tests "HD5 and HNP1-3" and "LE and HD5 and HNP1-3" also increased specificity but differed only slightly from "LE and HD5."

\section{DISCUSSION}

In this prospective study, we evaluated two AMPs as novel biomarkers for pediatric UTI. We demonstrated that both HD5 and HNP1-3 performed well as diagnostic tests to predict positive urine culture in children, with areas under the ROC curves of 0.86 and 0.88 for HD5 and HNP1-3, respectively. For test comparisons, we sought to determine whether combinations of highly sensitive tests would increase specificity without decreasing sensitivity. When compared to LE alone, the combination test "LE and HD5" increased specificity by $6 \%$ in all patients and $13 \%$ in the clean-catch subgroup without affecting sensitivity. The addition of HNP1-3 to LE did not improve specificity, but the combination test "HD5 and HNP1-3" did increase specificity by $12 \%$ in the clean-catch subgroup. The combination test "LE and HD5 and HNP1-3" also increased specificity in all patients and the clean-catch subgroup, but the results were only slightly better than "LE and HD5" without HNP1-3. These results suggest that urine AMPs have potential to improve standard methods for diagnosing UTI in children.

The rationale for identifying novel UTI biomarkers stems from the limited utility of currently available point-of-care tests for the diagnosis of UTI in children. The high specificity (99\%) but low sensitivity (45\%) of urinary nitrite in our cohort is comparable to results of prior studies (5-8). Thus, clinicians can expect a positive urine culture when nitrite is detected, but a negative nitrite test result does not rule out a UTI. Regarding LE, three pediatric meta-analyses and one recent, large study reported sensitivities of $72-83 \%$ and specificities of $78-87 \%$ (5-8). In our study, LE sensitivity (97\%) was greater than results of prior studies, while specificity (75\%) was comparable. A high false-positive rate of LE may lead to overdiagnoses and unnecessary antibiotic exposure. False-positive LE tests occur because pyuria is associated with a number of other conditions including acute febrile illnesses, urinary calculi, sexually transmitted infections, intrinsic renal diseases, and other disorders (15). Congruent results of LE and nitrite (both positive or both negative) help to rule in or rule out a UTI $(16,17)$, but the common scenario in which LE is positive 
a

\begin{tabular}{|c|c|c|c|c|c|c|c|}
\hline Test & Sensitivity, \% & Specificity, \% & \multicolumn{4}{|c|}{ Change in Specificity, \% (95\% Cl) } & $P$ value \\
\hline LE $\geq$ trace & 97 & 75 & \multicolumn{3}{|c|}{ reference value } & \multirow[b]{2}{*}{$-10(-17$ to -3$)$} & \multirow[b]{2}{*}{0.006} \\
\hline HD5 > $174 \mathrm{pg} / \mathrm{mgCr}$ & 100 & 65 & & 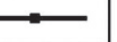 & & & \\
\hline HNP1-3 > 384 pg/mgCr & 97 & 65 & & $\longrightarrow$ & & $-10(-14$ to -4$)$ & $<0.001$ \\
\hline LE and HD5 & 97 & 81 & & & $\rightarrow-$ & $+6(+3$ to +10$)$ & $<0.001$ \\
\hline LE and HNP1-3 & 97 & 76 & & & - & $+1(0$ to +3$)$ & 0.50 \\
\hline HD5 and HNP1-3 & 97 & 76 & & & $=$ & $+1(-4$ to +7$)$ & 0.67 \\
\hline \multirow[t]{3}{*}{ LE and HD5 and HNP1-3 } & 97 & 82 & & & $\rightarrow$ & $+7(+3$ to +11$)$ & $<0.001$ \\
\hline & & -30 & -20 & -10 & $10 \quad 20$ & 30 & \\
\hline & \multicolumn{4}{|c|}{ Decreased specificity } & \multicolumn{2}{|c|}{ Increased specificity } & \\
\hline
\end{tabular}

b

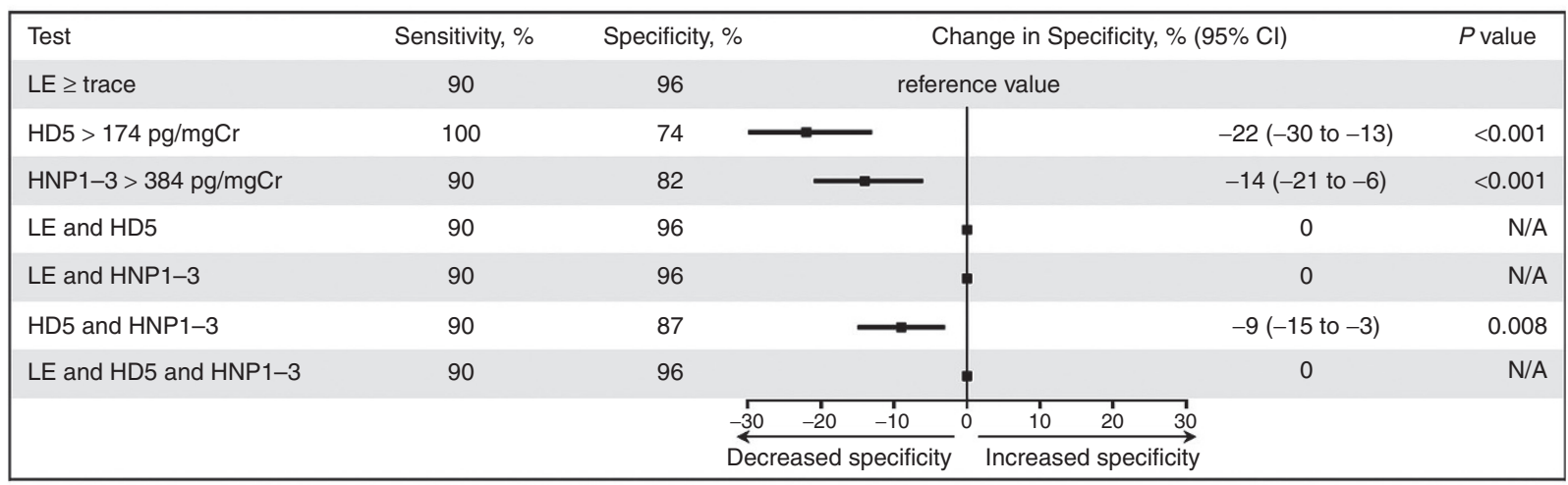

c

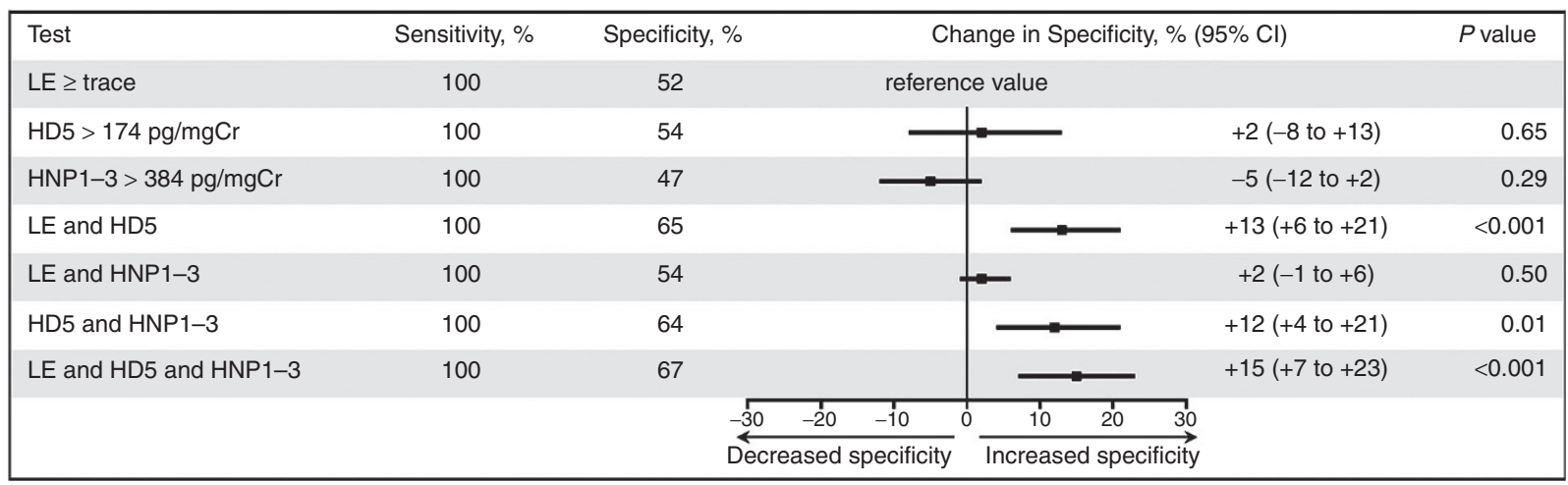

Figure 2. Comparisons of sensitivity and specificity of LE, HD5, and HNP1-3, alone and in combination. (a) All study patients. (b) Catheterization subgroup. (c) Clean-catch subgroup. Graphs display the change in specificity of each individual or combination test compared to LE $\geq$ trace. Horizontal bars represent $95 \%$ confidence intervals. HD5, $\alpha$-defensin 5; HNP1-3, human neutrophil peptides 1-3; LE, leukocyte esterase; N/A, not applicable.

but nitrite is negative creates diagnostic uncertainty. Improved rapid tests are needed to aid the accurate diagnosis of UTI.

In our study, both HD5 and HNP1-3 demonstrated potential to improve UTI diagnostic accuracy. Our results show that HD5 and HNP1-3 are induced during UTI. The higher concentrations of HD5 and HNP1-3 in culture-positive than culture-negative urine samples in our study is consistent with prior investigations performed in small numbers of children and adults $(12,13)$. The areas under the ROC curves for HD5 and HNP1-3 were between 0.75 and 0.9 and thus generally indicate "good" overall diagnostic value of the biomarkers (18). However, HNP1-3 did not improve specificity when combined with LE, perhaps because LE and HNP1-3 are both neutrophil markers and therefore indicate pyuria. In contrast, HD5 is expressed throughout the urothelium of the lower urinary tract and in the nephron and collecting tubules of the kidney (12). As an epithelial-derived AMP, HD5 likely performed well as a UTI biomarker independent of pyuria. Indeed, we found that the addition of HD5 to LE improved specificity. Still, there were some false-positive HD5 test results. We speculate that reasons for false-positive HD5 tests may include urethritis, viral cystitis, UTI due to pathogens that do not grow well in culture, noninfectious inflammatory conditions of the kidneys and urinary tract, and possibly other currently unrecognized 
stimuli for HD5 expression in the urine. Additionally, contamination of urine specimens with vaginal secretions could potentially result in false-positive HD5 test results because HD5 is produced in the female genital tract (11).

Novel UTI biomarkers may provide more benefit in certain clinical scenarios than others. In our study, the specificity of LE was much lower in the clean-catch subgroup. The data does not differentiate whether the difference in specificity was, in full or in part, due to the method of collection. Age, toilet-training status, or combinations of factors may have accounted for the difference. We are not aware of previous studies that directly compared LE specificity in samples collected by catheterization vs. a clean-catch method. A previous study did compare characteristics of the urine dipstick by age group and found lower specificity of the combination test "LE or nitrite" in children aged $\geq 2$ y (of whom only $10 \%$ were catheterized) than children aged $<2$ y (of whom $88 \%$ were catheterized) (19). Whatever the reasons for the difference, in our study, the greatest improvement in specificity was observed in the clean-catch subgroup, in whom combination tests "LE and HD5," "HD5 and HNP1-3," and "LE and HD5 and HNP1-3" all increased the specificity by $>10 \%$ compared to LE alone. Clinically, fewer false-positive tests may translate to decreased unnecessary empiric antibiotic therapy for patients evaluated for a UTI.

Our study has some limitations. First, we included all patients in whom urine testing was performed at the discretion of the treating clinician, irrespective of the pretest probability for UTI. Although the majority of the study population had at least one sign or symptom potentially suggestive of a UTI, perhaps test characteristics would differ in a cohort limited to only those patients with a high pretest probability (20). Next, two urinalysis machines were used in our study, depending on whether the dipstick analysis was performed in the ED/UC laboratories or the hospital laboratory. Dipstick results may differ at other centers that use different urinalysis machines. Last, the definition for a positive urine culture has not been established for all age groups and methods of urine collection. We chose a single definition for all patients based on current studies and guidelines (6,21-23).

In summary, we found that urine HD5 and HNP1-3 concentrations performed well as biomarkers for predicting positive urine culture in children. When combined with LE, HD5 provided the greatest increase in specificity without decreasing sensitivity, particularly when a clean-catch method of urine collection was used. We conclude that urine AMP profiles are a promising novel strategy as an adjunct to dipstick urinalysis to aid diagnosis and guide empiric management when UTI is suspected. Future research is needed to evaluate additional AMPs that may provide further improvements in specificity and/or sensitivity. Also, determination of AMP performance in subgroups of patients, such as those with neurogenic bladder and complicated urinary tracts, warrants investigation. Finally, development of rapid assays for urine AMP concentrations will ultimately be necessary to evaluate them as novel biomarkers at the point of care.

\section{METHODS}

\section{Study Enrollment}

Children were prospectively enrolled in the ED and main campus UC at Nationwide Children's Hospital, Columbus, Ohio, from 20 November 2013 to 2 July 2014 . Patients aged $\leq 18$ y who met the following inclusion criteria were enrolled during times when research staff was available: (i) both dipstick urinalysis and urine culture were performed for any clinical indication and (ii) excess urine sample was available. Patients were excluded if (i) they had received antibiotics in the $7 \mathrm{~d}$ before presentation to the ED or UC or (ii) the urine sample collected had insufficient volume for performance of all investigational assays. The institutional review board at Nationwide Children's Hospital approved the study and granted a waiver of informed consent (IRB13-00090).

\section{Clinical Data Collection and Analysis}

Electronic medical records were reviewed for pertinent demographic, clinical, and laboratory data, including urine dipstick and urine culture results. Fever was considered present when reported by the patient or caregiver or when a temperature $\geq 38^{\circ} \mathrm{C}$ was documented in the ED/UC. Dipstick urinalyses were performed either in the ED or UC laboratory using the CLINITEK Status + Analyzer (Siemens, Tarrytown, NY) or in the hospital chemistry laboratory using the iChem Velocity instrument (Iris Diagnostics, Chatsworth, CA). Urine was plated by clinical microbiology laboratory staff using calibrated loops and cultured on 5\% sheep blood and MacConkey agar biplates and incubated at $35^{\circ} \mathrm{C}$ in ambient atmosphere. A positive urine culture result consisted of $\geq 50,000$ colony-forming units per milliliter of a single uropathogen $(21,23)$. Urine cultures were considered negative if they yielded $<50,000$ colony-forming units $/ \mathrm{ml}$, mixed bacteria, or likely contaminants such as Lactobacillus species, coagulase-negative staphylococci, or Corynebacterium species (23).

\section{Sample Collection}

After ensuring sufficient urine volume was available for clinical diagnostic tests, excess urine was immediately collected in AssayAssure urine collection tubes (Thermo Scientific, Waltham, MA) containing a bacteriostatic preservative that suppresses nuclease and protease activity and preserves urine specimens at room temperature for up to $26 \mathrm{~d}$ according to the manufacturer.

\section{AssayAssure Validation}

We independently verified the stability of AMPs for up to $14 \mathrm{~d}$ in AssayAssure preservative by measuring serial urine concentrations of ribonuclease 7 (RNase 7). We chose to measure RNase 7 because it is an AMP that is constitutively expressed in the urine of healthy individuals. On day 0, urine samples from two healthy individuals were collected and stored in AssayAssure urine collection tubes. On days 1, 2, 7 , and 14 , an aliquot of each sample was removed and stored at $-80^{\circ} \mathrm{C}$ until analyzed. After the 14-d period, all frozen samples were analyzed simultaneously in a single enzyme-linked immunosorbent assay to determine the concentrations of RNase 7, as previously described (24). Concentrations of RNase 7 remained stable in AssayAssure during the 14 -d validation period (Supplementary Figure S1 online).

\section{Sample Processing and Analysis}

Within $7 \mathrm{~d}$ of collection, study samples were centrifuged at 3,000 rpm for $5 \mathrm{~min}$. The supernatant was saved in 300 to $500 \mu \mathrm{l}$ aliquots and stored at $-80^{\circ} \mathrm{C}$ until analyzed. To evaluate subgroups by urine collection method, nearly equal numbers of study samples obtained by catheterization and clean-catch method were analyzed. Urine concentrations of HD5 and HNP1-3 were measured in duplicate by enzymelinked immunosorbent assay using commercial kits according to the manufacturers' instructions (HD5: Uscn Life Science, Wuhan, Hubei, China; HNP1-3: Hycult Biotech, Plymouth Meeting, PA). Study samples with concentrations less than the lower limit of quantification for HD5 or HNP1-3 were assigned a value of one-half the lower limit of quantification as calculated on a $\log _{10}$ curve (25). To account for urine dilution, concentrations of HD5 and HNP1-3 were normalized to urine creatinine concentration, which was measured by a colorimetric assay (Oxford Biomedical Research, Oxford, MI). 


\section{Sample Size Determination}

To design a study with $80 \%$ power, 176 total patients was adequate to ascertain a $10 \%$ difference in specificity between two diagnostic tests with a Type I error of 0.05 (26).

\section{Statistical Analysis}

Urine culture was the reference standard for evaluating test characteristics of the urine dipstick and AMPs. First, urine concentrations of each AMP were compared in culture-negative vs. culture-positive urine samples using the Mann-Whitney rank-sum test (non-normally distributed, continuous variables). Next, a ROC curve was generated for each AMP, and the area under the curve was calculated using the scientific software GraphPad Prism 6 (GraphPad Software, La Jolla, CA). Optimal threshold values for positive HD5 and HNP1-3 test results were modeled to maximize specificity while ensuring sensitivity no less than that of LE. Last, the sensitivities and specificities of LE, HD5, HNP1-3, and combinations of the aforementioned tests were compared. Test combinations using the conjunction "and" were considered positive only if all components were positive. Test combinations using the conjunction "or" were considered positive if any individual component was positive. Comparisons between subgroups utilized Fisher's exact test (categorical variables) or Mann-Whitney rank-sum test (non-normally distributed, continuous variables). McNemar's test was used to evaluate differences in specificity between two diagnostic tests. All $P$ values were two sided.

\section{SUPPLEMENTARY MATERIAL}

Supplementary material is linked to the online version of the paper at http:// www.nature.com/pr

\section{ACKNOWLEDGMENTS}

The authors thank the Nationwide Children's Hospital Emergency Department Research Coordinators for enrolling patients in the study and collecting urine samples, and Evan Barr-Beare, Research Assistant at Nationwide Children's Hospital, for assisting with the urine biorepository management. This study was presented in part as a platform presentation at the Pediatric Academic Societies' Meeting, 30 April-3 May 2015, San Diego, CA.

\section{STATEMENT OF FINANCIAL SUPPORT}

All phases of this study were supported by Intramural Grant 201213, The Research Institute at Nationwide Children's Hospital, Columbus, OH, and Intramural Grant 20053814, Infectious Diseases Consortium, The Research Institute at Nationwide Children's Hospital, Columbus, OH. Statistical analysis also was supported by Award Number Grant UL1TR001070 from the National Center for Advancing Translational Sciences. The content is solely the responsibility of the authors and does not necessarily represent the official views of the National Center for Advancing Translational Sciences or the National Institutes of Health, Bethesda, MD. J.D.S. is supported by the National Institute of Health Grant (NIDDK) K08 DK094970-03, Bethesda, MD.

Disclosure: The authors have no conflicts of interest or relevant financial relationships related to this manuscript to disclose.

\section{REFERENCES}

1. Copp HL, Shapiro DJ, Hersh AL. National ambulatory antibiotic prescribing patterns for pediatric urinary tract infection, 1998-2007. Pediatrics 2011;127:1027-33.

2. Freedman AL; Urologic Diseases in America Project. Urologic diseases in North America Project: trends in resource utilization for urinary tract infections in children. J Urol 2005;173:949-54.

3. Spencer JD, Schwaderer A, McHugh K, Hains DS. Pediatric urinary tract infections: an analysis of hospitalizations, charges, and costs in the USA. Pediatr Nephrol 2010;25:2469-75.

4. Shaikh N, Morone NE, Lopez J, et al. Does this child have a urinary tract infection? JAMA 2007;298:2895-904.

5. Downs SM. Technical report: urinary tract infections in febrile infants and young children. The Urinary Tract Subcommittee of the American
Academy of Pediatrics Committee on Quality Improvement. Pediatrics 1999;103:e54.

6. Kazi BA, Buffone GJ, Revell PA, Chandramohan L, Dowlin MD, Cruz AT. Performance characteristics of urinalyses for the diagnosis of pediatric urinary tract infection. Am J Emerg Med 2013;31:1405-7.

7. Williams GJ, Macaskill P, Chan SF, Turner RM, Hodson E, Craig JC. Absolute and relative accuracy of rapid urine tests for urinary tract infection in children: a meta-analysis. Lancet Infect Dis 2010;10:240-50.

8. Gorelick MH, Shaw KN. Screening tests for urinary tract infection in children: a meta-analysis. Pediatrics 1999;104:e54.

9. Spencer JD, Schwaderer AL, Becknell B, Watson J, Hains DS. The innate immune response during urinary tract infection and pyelonephritis. Pediatr Nephrol 2014;29:1139-49.

10. Jones DE, Bevins CL. Paneth cells of the human small intestine express an antimicrobial peptide gene. J Biol Chem 1992;267:23216-25.

11. Quayle AJ, Porter EM, Nussbaum AA, et al. Gene expression, immunolocalization, and secretion of human defensin-5 in human female reproductive tract. Am J Pathol 1998;152:1247-58.

12. Spencer JD, Hains DS, Porter E, et al. Human alpha defensin 5 expression in the human kidney and urinary tract. PLoS One 2012;7:e31712.

13. Ihi T, Nakazato M, Mukae H, Matsukura S. Elevated concentrations of human neutrophil peptides in plasma, blood, and body fluids from patients with infections. Clin Infect Dis 1997;25:1134-40.

14. Tikhonov I, Rebenok A, Chyzh A. A study of interleukin-8 and defensins in urine and plasma of patients with pyelonephritis and glomerulonephritis. Nephrol Dial Transplant 1997;12:2557-61.

15. Dieter RS. Sterile pyuria: a differential diagnosis. Compr Ther 2000;26:150-2.

16. Mori R, Yonemoto N, Fitzgerald A, Tullus K, Verrier-Jones K, Lakhanpaul M. Diagnostic performance of urine dipstick testing in children with suspected UTI: a systematic review of relationship with age and comparison with microscopy. Acta Paediatr 2010;99:581-4.

17. Whiting P, Westwood M, Watt I, Cooper J, Kleijnen J. Rapid tests and urine sampling techniques for the diagnosis of urinary tract infection (UTI) in children under five years: a systematic review. BMC Pediatr 2005;5:4.

18. Ray P, Le Manach Y, Riou B, Houle TT. Statistical evaluation of a biomarker. Anesthesiology 2010;112:1023-40.

19. Shaw KN, Hexter D, McGowan KL, Schwartz JS. Clinical evaluation of a rapid screening test for urinary tract infections in children. J Pediatr 1991;118:733-6.

20. Lachs MS, Nachamkin I, Edelstein PH, Goldman J, Feinstein AR, Schwartz JS. Spectrum bias in the evaluation of diagnostic tests: lessons from the rapid dipstick test for urinary tract infection. Ann Intern Med 1992;117:135-40.

21. Hoberman A, Wald ER, Reynolds EA, Penchansky L, Charron M. Pyuria and bacteriuria in urine specimens obtained by catheter from young children with fever. J Pediatr 1994;124:513-9.

22. Pryles CV, Steg NL. Specimens of urine obtained from young girls by catheter versus voiding; a comparative study of bacterial cultures, gram stains and bacterial counts in paired specimens. Pediatrics 1959;23:441-52.

23. Roberts KB, Subcommittee on Urinary Tract Infection, Steering Committee on Quality Improvement and Management. Urinary tract infection: clinical practice guideline for the diagnosis and management of the initial UTI in febrile infants and children 2 to 24 months. Pediatrics 2011;128:595-610.

24. Spencer JD, Schwaderer AL, Dirosario JD, et al. Ribonuclease 7 is a potent antimicrobial peptide within the human urinary tract. Kidney Int 2011;80:174-80.

25. Coady K, Marino T, Thomas J, Sosinski L, Neal B, Hammond L. An evaluation of 2,4-dichlorophenoxyacetic acid in the Amphibian Metamorphosis Assay and the Fish Short-Term Reproduction Assay. Ecotoxicol Environ Saf 2013;90:143-50.

26. Connor RJ. Sample size for testing differences in proportions for the paired-sample design. Biometrics 1987;43:207-11. 